\title{
Urinary proteome analysis and the management of ureteropelvic junction obstruction
}

\author{
Hrair-George O. Mesrobian
}

Received: 24 December 2009/Revised: 12 March 2010 /Accepted: 15 March 2010/Published online: 21 April 2010

(C) IPNA 2010

\begin{abstract}
Ureteropelvic junction obstruction (UPJO) detected prenatally may over time deteriorate and require surgery, improve, or remain stable, and it may take upwards of 3 years for its natural history to unfold. Clinical decisions for or against operative corrections are usually based on scintigraphy followup studies. A non-invasive method for facilitating clinical decisions has recently been presented: urinary proteome analysis utilizing capillary electrophoresis mass spectrometry (CE-MS) has been shown to predict the outcome of UPJO in newborns. The group that developed this assay has now validated their seminal findings and extended the investigations to older age groups (this issue). The results of the blinded analysis correctly identified patients with UPJO who underwent surgery with a sensitivity of $83 \%$ (5 of 6 patients) and a specificity of $92 \%$ (12 of 13 patients) in infants up to 1 year of age. The validity of the analysis was poor in children $>1$ year of age with unilateral UPJO. A large number of patients will be needed to answer the question of to what extent the normal variability of urinary proteomes overlap with the variability of the pattern in UPJO beyond early infancy.
\end{abstract}

Keywords Hydronephrosis · Prognostic biomarkers ·

Ureteropelvic junction obstruction .

Urinary proteome analysis

The evaluation and management of newborns and infants with ureteropelvic junction obstruction (UPJO) continues to evolve. Most cases of UPJO are detected prenatally by

H.-G. O. Mesrobian ( $\square)$

Department of Urology,

Medical College and Children's Hospital of Wisconsin, 999 N92nd Street, Suite C 330,

Milwaukee, WI 53226, USA

e-mail: hmesrobi@mcw.edu virtue of maternal sonography. Once confirmed by postnatal sonography, the main challenge resides in identifying early on in infancy the $20-30 \%$ of children in whom the disease is likely to progress over time and require surgery [1]. It is believed that the majority of infants are asymptomatic, and it may take upwards of 3 years for a kidney to declare itself. In observational studies, the disease has been shown to resolve spontaneously over time in an ill-defined but well-documented group of patients [2]. In the majority of infants, imaging or clinical parameters do not discriminate between those who are likely to progress and those who are not. In a landmark study, Decramer et al. demonstrated that urinary proteome profiles identified by capillary electrophoresis coupled to mass spectrometry (CEMS) can be used both to discriminate between patients with unilateral UPJO and normal individuals and to identify UPJO patients whose disease is likely to "progress over time" [3]. These prognostic biomarkers may assist the clinician in the identification and early diagnosis of the subgroup of patients from the outset in whom the disease is likely to progress and early surgery is advisable while diminishing the frequency of imaging and follow-ups in the remainder [4].

Urinary proteome analysis has been a rapidly growing discipline, with applications in biomedical research aimed at the discovery of disease biomarkers, therapeutic drug targets, and disease mechanisms [5]. Validation of new modalities, such as this one, is the first step towards generalizability and clinical applicability.

Drube et al. (this issue [6]) from the Department of Nephrology at Children's Hospital in Hannover obtained urinary specimens from patients with various grades of UPJO during the course of routine clinical care and shipped them to a commercial diagnostic company which distributes the test originally described by Decramer et al. [3]. In six patients $<1$ year of age, the CE-MS analysis correctly 
classified five as needing surgery based on diuresis renography criteria, thereby confirming the validity of this approach in this pilot project. In patients $>1$ year of age at the time of analysis, the results from the CE-MS analysis and diuresis renography were concordant only in one of five patients. The test correctly predicted the absence of surgical disease in 12 of 13 patients in the $<1$ year age group and only in one of five patients in the $>1$ year age group. We should note that the diuretic renogram using J-123-Hippuran provided the basis for surgery, namely, failure of the tracer activity to drop by at least $50 \%$ within $20 \mathrm{~min}$ of the administration of the diuretic ( $1 \mathrm{mg} / \mathrm{kg}$ of furosemide).

This article demonstrates that - in contrast to other body fluids or tissues - urinary proteins remain stable, thus allowing the performance of reliable CE-MS analysis. A major advantage to this approach over historical methods resides in the fact that it allows for the identification of a number of proteins and peptides as opposed to a single biomarker which may differ between a disease state (UPJO) and normal. This difference represents a major improvement because a single gene, protein, or molecule may not by itself fulfill all of the properties of a disease biomarker. Many different biologic processes come into play, including compensatory and adaptive mechanisms, in response to a lesion such as UPJO with early onset during development. A biomarker discovery tool, such as CE-MS, is more suited for capturing the sum total of perturbations which occur in the diseased compartment and are reflected in the bladder urine specimens. These changes are captured as differences in the urinary protein profile relative to that of age-matched normal individuals. The commercial lab was blinded to the degree of hydronephrosis, which was classified as either obstructive or non-obstructive based on the diuresis renography results.

Although the authors differentiate between age groups $(<1$ or $>1$ years), we note that three of the five patients in the $>1$ year age group were being followed for known UPJO. Without knowledge of their urinary proteome profile at an earlier age, caution is recommended in drawing conclusions regarding sensitivity and specificity in this older age group. It is well known that normal individuals display a great deal of biologic variability, which is reflected in the urinary proteome profile [7]. In addition, the hydronephrotic kidney in one of the patients in this older age group did have reduced function $(23 \%)$. It is not known how kidney function reductionfrom any cause - is translated into the proteome pattern: are the observed changes specific to the disease (UPJO) or nonspecific and reflect reduced glomerular and tubular function? Nevertheless, the validity of this approach is demonstrated in the $<1$ year age group with a high sensitivity and, more importantly, high specificity $(92 \%)$. The latter is desirable because it protects against unnecessary operations if CE-MS analysis were the only criterion for recommending surgical repair.
Finally, although the authors correctly state that the threshold for defining the presence of obstruction on the diuretic renography is arbitrary, they utilize it as the gold standard against which the CE-MS findings are validated. This is somewhat of a circular argument where the evidence for the proposition contains the proposition itself. To get around this predicament, it may be desirable to initially follow UPJO patients over time non-operatively and monitor the individual split renal function and degree of dilatation (for example, by measuring the antero-posterior diameter of the hydronephrotic renal pelvis). Surgery would be indicated for deterioration (for example, a $\geq 10 \%$ decrease in individual renal function or a $\geq 10 \%$ increase in the antero-posterior diameter). The urinary proteome profile obtained at the time of surgery may then contain the elusive "gold standard" definition of obstruction.

In conclusion, the article by Drube et al. [6] validates the proteomic approach in a small number of patients with unilateral UPJO in whom the analysis is performed at $<1$ year of age. It is not clear from this study why the sensitivity deteriorates in patients $>1$ year of age. Could the normal variability of the urinary proteome in the older age group (with the hydronephrotic kidney having completed its development despite the presence of UPJO) overlap with the variability in the disease, resulting in non-discrimination? These important questions can be answered with continued investigation in a large number of patients emanating from multiple centers. The stability and the easy availability of urinary specimens constitute powerful arguments in favor of continued research in this and related fields.

\section{References}

1. Dhillon HK (1998) Prenatally diagnosed hydronephrosis: The Great Ormond Street Experience. Br J Urol 2:39-44

2. Ulman I, Jayanthi VR, Koff SA (2000) The long-term follow-up of newborns with severe unilateral hydronephrosis initially treated nonoperatively. J Urol 164:1101-1104

3. Decramer S, Wittke S, Mischak H, Zürbig P, Walden M, Bouissou F, Bascands JL, Schanstra JP (2006) Predicting the clinical outcome of congenital unilateral ureteropelvic junction obstruction in newborn by urinary proteome analysis. Nat Med 12:398-400

4. Mesrobian HG (2009) The value of newborn urinary proteome analysis in the evaluation and management of ureteropelvic junction obstruction: a cost-effectiveness study. World J Urol 27:379-383

5. Thongboonkerd V (2010) Current status of renal and urinary proteomics: ready for routine clinical application? Nephrol Dial Transplant 25:11-16

6. Drube J, Zürbig P, Schiffer E, Lau E, Ure B, Glüer S, Kirschstein M, Pape L, Decramer S, Bascands J, Schanstra JP, Mischak H, Ehrich JH (2010) Urinary proteome analysis identifies infants but not older children requiring pyeloplasty. Pediatr Nephrol. doi:10.1007/s00467010-1455-8

7. Little PF, Williams RB, Wilkins MR (2008) Inter-individual variation in expression: a missing link in biomarker biology? Trends Biotechnol 27:5-10 